\title{
Insulin Therapy in Burn Patients Does not Contribute to Hepatic Triglyceride Production
}

\author{
Asle Aarsland, ${ }^{\circ}$ David L. Chinkes, ${ }^{*}$ Yoichi Sakurai, ${ }^{*}$ Thuan T. Nguyen, ${ }^{*}$ David N. Herndon, ${ }^{\star}$ and Robert R. Wolfe ${ }^{\star \ddagger}$ \\ The Metabolism Unit, Shriners Burns Institute, *Department of Surgery and ${ }^{\ddagger}$ Department of Anesthesiology, University of Texas Medical \\ Branch, Galveston, Texas 77550-1220
}

\begin{abstract}
Lipid kinetics were studied in six severely burned patients who were treated with a high dose of exogenous insulin plus glucose to promote protein metabolism. The patients were $20 \pm 2$-yr-old (SD) with $63 \pm 8 \%$ total body surface area burned. They were studied in a randomized order $(a)$ in the fed state on the seventh day of a control period (C) of continuous high-carbohydrate enteral feeding alone, and $(b)$ on the seventh day of enteral feeding plus exogenous insulin ( $200 \mathrm{pmol} / \mathrm{h}=28 \mathrm{U} / \mathrm{h}$ ) with extra glucose given as needed to avoid hypoglycemia $(I+G)$. Despite a glucose delivery rate $\sim 100 \%$ in excess of energy requirements, the following lipid parameters were unchanged: (a) total hepatic VLDL triglyceride (TG) secretion rate $(0.165 \pm 0.138[C]$ vs. $0.154 \pm$ $\left.0.138 \mathrm{mmol} / \mathrm{kg} \cdot \mathrm{d}^{-1}[\mathrm{I}+\mathrm{G}]\right)$, (b) plasma TG concentration $(1.58 \pm 0.66[\mathrm{C}]$ vs. $1.36 \pm 0.41 \mathrm{mmol} / \mathrm{liter}[\mathrm{I}+\mathrm{G}])$, and $(c)$ plasma VLDL TG concentration $(0.68 \pm 0.79[\mathrm{C}]$ vs. $0.67 \pm$ $0.63 \mathrm{mmol} /$ liter $[\mathrm{I}+\mathrm{G}])$. Instead, the high-carbohydrate delivery in conjunction with insulin therapy increased the proportion of de novo-synthesized palmitate in VLDL TG from $13 \pm 5 \%$ (C) to $34 \pm 14 \%(\mathrm{I}+\mathrm{G})$, with a corresponding decreased amount of palmitate from lipolysis. In association with the doubling of the secretion rate of de novo-synthesized fatty acid (FA) in VLDL TG during insulin therapy $(P>0.5)$, the relative amount of palmitate and stearate increased from $35 \pm 5$ to $44 \pm 8 \%$ and $4 \pm 1$ to $7 \pm 2 \%$, respectively, in VLDL TG, while the relative concentration of oleate and linoleate decreased from $43 \pm 5$ to $37 \pm 6 \%$ and $8 \pm 4 \%$ to $2 \pm 2 \%$, respectively. A 15 -fold increase in plasma insulin concentration did not change the rate of release of FA into plasma $\left(8.22 \pm 2.86[\mathrm{C}] \mathrm{vs} .8 .72 \pm 6.68 \mathrm{mmol} / \mathrm{kg} \cdot \mathrm{d}^{-1}\right.$ $[\mathrm{I}+\mathrm{G}]$. The peripheral release of $\mathrm{FA}$ represents a far greater potential for hepatic lipid accumulation in burn patients than the endogenous hepatic fat synthesis, even during excessive carbohydrate intake in conjunction with insulin therapy. (J. Clin. Invest. 1998. 101:2233-2239.) Key words: fat synthesis $\bullet$ lipogenesis $\bullet$ lipolysis $\bullet$ carbohydrate $\bullet$ liver
\end{abstract}

Address correspondence to Dr. Robert R. Wolfe, Shriners Burns Institute, 815 Market Street, Galveston, TX 77551. Phone: 409-7706605; FAX: 409-770-6825; E-mail: rwolfe@sbi.utmb.edu

Received for publication 21 March 1997 and accepted in revised form 26 February 1998.

J. Clin. Invest.

(C) The American Society for Clinical Investigation, Inc. 0021-9738/98/05/2233/07 \$2.00

Volume 101, Number 10, May 1998, 2233-2239

http://www.jci.org

\section{Introduction}

The primary goal of the nutritional/metabolic management of burn patients is to promote wound healing and resistance to infection while preventing persistent loss of muscle protein (1). Since hepatic fat deposition is commonly associated with burn injury (2-7) the management should also be aimed at preserving the integrity of the liver. The limited effectiveness of nutritional support alone in critically ill patients has led to the investigation of various pharmacological approaches to optimize the metabolic response $(8,9)$. We have recently reported that providing exogenous insulin may be a useful approach in preventing muscle wasting in severely burned patients by stimulating protein synthesis (10). To maintain euglycemia during insulin treatment it was necessary to give carbohydrates at a rate $\sim 100 \%$ in excess of their energy requirement. Using indirect calorimetry it has been previously shown that a comparable amount of excess carbohydrate intake is a significant stimulus for lipogenesis $(11,12)$. However, indirect calorimetry can only determine net lipogenesis at a whole body level, and does not provide information on the localization of the lipogenic process. Based on indirect evidence the liver was assumed until recently to be the main site for carbohydrate induced lipogenesis $(12,13)$. However, we have recently presented evidence suggesting that in healthy volunteers the liver only converts a minor fraction of surplus dietary carbohydrate into fat (14).

In this study we investigated the extent to which the administering of a high dose of insulin and glucose causes hepatic fat accumulation in burn patients. Insulin is a potent inhibitor of adipose tissue lipolysis (15) and could potentially decrease intrahepatic fatty acid (FA) ${ }^{1}$ availability for storage. On the other hand, insulin and glucose in combination can spare intrahepatic fat from oxidation $(16,17)$ and stimulate hepatic fat synthesis (18), both factors that can contribute to hepatic steatosis. Finally, while hepatic secretion of fat in the form of VLDL triglyceride (TG) is inhibited by short-term hyperinsulinemia (19), the effect of long-term hyperinsulinemia is more complex and could lead to stimulated secretion $(18,20)$. Thus, a combined insulin plus glucose treatment can potentially influence hepatic steatosis by changing the hepatic availability of both endogenously de novo-synthesized FA and of preformed FA from the adipose tissue and the diet. Therefore, in six severely burned patients, on the seventh day of a control period and on the seventh day of an exogenous insulin plus glucose treatment period, we measured the peripheral release of FA, and the VLDL TG bound secretion of de novo synthesized- and preformed FAs coming from the liver.

1. Abbreviations used in this paper: FA, fatty acid; RA, rate of appearance; TBSA, total body surface area; TG, triglyceride. 


\section{Methods}

Patients. Tracer infusion studies were performed in six patients who were admitted to the Burn Unit of The University of Texas Medical Branch and/or the Shriners Burns Institute in Galveston, Texas. Characteristics of the individual patients are presented in Table I. They ranged in age from 15 to $28 \mathrm{yr}$ (mean of $22 \pm 5 \mathrm{yr}$ ) and had a mean burn size of $62 \pm 25 \%$ of total body surface area (TBSA) (range, $35-90 \%$ TBSA), of which $38 \pm 32 \%$ were third degree (range, $5-90 \%$ TBSA). Three patients were diagnosed as having smoke inhalation injury of the respiratory tract. The exact date of the first study was determined by the first operative procedure. This varied between the 7th and 11th day after burn, depending on clinical factors such as hemodynamic and respiratory status.

Experimental design. Each patient was studied twice and, thus, served as their own control. In one study they were fed an enteral diet, in the other study, in addition to the enteral diet, they were coadministered insulin plus glucose. The carbohydrate-rich enteral diet (Vivonex ${ }^{\circledR}$ T.E.N.; Sandoz Nutrition Corp., Minneapolis, MN) was infused continuously via nasogastric and duodenal catheters, providing 1.4 times as many calories as the measured resting energy expenditure. The composition of the enteral diet was $15 \%$ of calories as free amino acids, $82 \%$ as carbohydrate, and $3 \%$ as fat. The concentration of Vivonex $(\mathrm{J} / \mathrm{ml})$ was individually adjusted so that there were no clinical symptoms such as diarrhea. The high-carbohydrate intake was used to diminish the amount of intravenously infused dextrose required to prevent hypoglycemia during the insulin infusion. A comparable low-fat/high-carbohydrate diet has been previously shown to eliminate the influx of exogenous fat via absorption (21) so that the FAs and the TG that appear in plasma under these dietary conditions are from endogenous sources. 6-h isotopic tracer infusion studies were performed during the continuous enteral infusion of the diet alone (control period) and during the continuous infusion of the enteral diet along with a continuous infusion of exogenous insulin (insulin plus glucose period). Insulin (Humulin R; Eli Lilly and Co., Indianapolis, IN) was infused continuously for $7 \mathrm{~d}$ through the central venous catheter to achieve a concentration of plasma insulin $(\sim 6,500$ pmol/liter $[\sim 900 \mu \mathrm{U} / \mathrm{ml}]$ ) designed to elicit the maximal response. The insulin infusion was started at a slow rate and was increased incrementally every $2 \mathrm{~h}$ until the final infusion rate of $200 \mathrm{pmol} / \mathrm{h}(\sim 28$ $\mathrm{U} / \mathrm{h}$ ) was achieved within $6 \mathrm{~h}$. Plasma glucose concentration was monitored hourly and exogenous glucose (2.78 M [50\%] dextrose) was infused as required to maintain euglycemia. The isotopic tracer infusion studies were performed at the end of each $7-\mathrm{d}$ treatment period. The exogenous glucose infusion rate was fixed during the 6-h isotope infusion study period. To avoid fluctuations in other parameters that may have affected metabolism due to the differences in the clinical course of the patients, the sequence of treatment periods was randomized. To coordinate each study with the clinical treatment, the first experimental period was started immediately after the first grafting procedure (after burn days 7-11). Therefore, the first tracer infusion study

\section{Table I. Patient Characteristics}

\begin{tabular}{lccc}
\hline \multicolumn{1}{c}{ Sex } & Age & Burn size & Burn size \\
\hline & $y r$ & $\%$ TBSA & $\% 3^{\circ}$ \\
M & 22 & 36 & 5 \\
M & 28 & 35 & 5 \\
M & 27 & 85 & 45 \\
F & 17 & 78 & 35 \\
M & 15 & 46 & 46 \\
M & 21 & 90 & 90 \\
Average & $22 \pm 5$ & $62 \pm 25$ & $38 \pm 32$ \\
\hline
\end{tabular}

was done $7 \mathrm{~d}$ after the first grafting procedure, immediately before the second grafting procedure. Thus, each tracer infusion was performed $7 \mathrm{~d}$ after a grafting procedure.

The patients received a $6-\mathrm{h}$ continuous $\left(2 \mu \mathrm{mol} / \mathrm{kg} \cdot \mathrm{min}^{-1}\right)$, nonprimed infusion of $\left[1,2-{ }^{13} \mathrm{C}\right]$ acetate $(99 \%$ enriched) (Isotec Inc., Miamisburg, $\mathrm{OH}$ ), and during the last hour of the infusion study a 1-h continuous $\left(0.04 \mu \mathrm{mol} / \mathrm{kg} \cdot \mathrm{min}^{-1}\right)$ infusion of $\left[1-{ }^{13} \mathrm{C}\right]$ palmitate $(99 \%$ enriched) (Cambridge Isotope Laboratories, Andover, MA). Both tracers were infused through a central venous catheter using calibrated syringe pumps (Harvard Apparatus, Natick, MA). Blood samples were drawn from the femoral artery catheters at $0,60,120,180$, $240,270,300,315,330,350,355,345$, and $360 \mathrm{~min}$ after the start of the isotopic tracer infusion and placed into $10-\mathrm{ml}$ vacutainers containing disodium EDTA for the determination of the isotopic enrichment of palmitate bound to VLDL TG and free plasma palmitate. All samples were placed on ice. Plasma was separated by centrifugation shortly after sampling. Samples for VLDL isolation were stored at $4^{\circ} \mathrm{C}$ until they were processed the next morning. All other samples were stored at $-20^{\circ} \mathrm{C}$ until they were processed. Blood samples for determination of insulin and glucagon concentration were collected in vacutainers containing EDTA. The tubes for glucagon analysis also contained aprotinin (Sigma Chemical Co., St. Louis, MO).

Analysis of samples. Plasma insulin and glucagon concentrations were measured by radioimmunoassay [(Incstar Corp., Stillwater, $\mathrm{MN}$ ), and (RSL glucagon kit; ICN Biomedicals, Inc., Irvine, CA) respectively]. Glucose and lactate concentrations were measured using an automated analyzer (Yellow Springs Instrument Inc., Yellow Springs, $\mathrm{OH})$.

VLDL was isolated from $3 \mathrm{ml}$ of plasma by overlaying the plasma with a density equal to 1.006 solution $(0.9 \% \mathrm{NaCl})$ and separated by spinning the samples in an ultracentrifuge (model L7-55; Beckman Instruments, Palo Alto, CA) in a 70.1 Ti rotor at 50,000 rpm $(171,500 \mathrm{~g}$ average) for $20 \mathrm{~h}$ at $15^{\circ} \mathrm{C}$. After ultracentrifugation, the VLDL TG was carefully removed along with the density solution found on top of the tube by the slicing tube technique. The total volume of the resulting VLDL suspension was $3 \mathrm{ml}$.

TG concentration in the VLDL suspension and in plasma was determined enzymatically (RA-500; Technicon Instruments Corp., Tarrytown, NY) (22). TG in the VLDL suspension was isolated by TLC, hydrolyzed to FFAs, and derivatized to FA methyl esters. FFAs were extracted from plasma, isolated by TLC, and derivatized to FA methyl esters. Palmitate and total FFA concentrations in plasma were determined by gas chromatography (model 5890; Hewlett-Packard Co., Palo Alto, CA) using heptadecanoic acid as an internal standard. The relative concentrations of individual FAs in the VLDL fraction were determined similarly by gas chromatography. Isotopic enrichment of palmitate was determined by gas chromatography-mass spectrometry (GCMS) (model 5992; Hewlett-Packard Co.) in the electron impact ionization mode for the ultimate computation of the tracer/tracee ratio. For the palmitate methyl ester the ions of mass-to-charge ratio (m/e) 270, 271, 272, 273, and 274 were selectively monitored.

Calculations. Sample analysis indicated that a physiological and isotopic steady-state existed during the infusion period in all studies, meaning that neither the concentration nor the enrichment changed during the time in which the samples were obtained. The rate of appearance of palmitate and the rate of secretion of VLDL-bound palmitate were calculated with use of steady-state equations (23). The total rate of appearance (RA) of FAs into the systemic circulation was calculated by dividing the rate of appearance of palmitate by the ratio of the concentrations of palmitate to total FFAs. This measurement of the rate of release of FA is not necessarily the same as the rate of lipolysis, because some FAs can be reesterified within the adipose tissue.

VLDL TGs secreted by the liver are principally derived from two sources of FA, (a) FAs that are synthesized de novo in the liver and (b) preformed FAs (18). The term preformed FAs is used collectively for FAs that arise from any other source than de novo synthesis. Potential sources of preformed FAs include the plasma FA from periph- 
eral lipolysis (adipose tissue), FA from lipolysis of lipoprotein remnants, or FA resulting from hydrolysis of intrahepatic TG. In our experiment, fat was omitted from the diet so that the plasma FFA was entirely derived from lipolysis. Our method of measuring the kinetics of VLDL-bound TG takes advantage of the fact that acetyl-CoA is the precursor for de novo FA synthesis. Labeled acetate, mixed with the endogenous pool of acetyl-CoA, labels newly formed FAs in the liver. A certain fraction of these labeled FAs is secreted from the liver as VLDL-bound FA. If the enrichment of the precursor pool is known, then the rate of secretion of de novo-synthesized FA can be calculated by measuring the rate of incorporation of the label into the product. The enrichment of the hepatic precursor pool for FA synthesis cannot be measured directly or even estimated in vivo. Therefore, the novelty of the current method is the examination of precursor enrichment based on the enrichment pattern (isotopomer distribution) of the product (VLDL-bound FA). The deduced precursor enrichment enables us to measure not only the rate of secretion of de novosynthesized palmitate, but also the total rate of secretion of the VLDL-bound palmitate based on the dilution principle. The rate of secretion of preformed palmitate is calculated by subtracting the rate of secretion of de novo-synthesized palmitate from the total rate of secretion of the FA. In this study, kinetics for palmitate are reported because palmitate is the predominate product of de novo fat synthesis and also serves as a representative FA for the preformed FAs. The total rate of VLDL TG secretion is obtained by dividing the total rate of palmitate secretion by the percentage of VLDL-bound palmitate. The specific formulas used to calculate the intrahepatic precursor enrichment for FA synthesis, the rate of secretion of de novo-synthesized palmitate bound to VLDL-TG, total rate of VLDL bound palmitate secretion, and the total rate of VLDL TG secretion has been reported previously (18).

Statistical analysis. Statistical comparisons between the control and insulin infusion periods were made using the paired $t$ test (24). $P<0.05$ was considered significant. All values in the text, tables, and figures are expressed as mean $\pm \mathrm{SD}$.

\section{Results}

Initially eight patients were enrolled in this study. Because of technical problems with two of the infusion studies, unrelated to patient care, two patients were excluded from the study. Thus, in a randomized, crossover study six burn patients (Table I) were treated with insulin at doses of $56.1 \pm 5.0 \mathrm{pmol} /$ $\mathrm{kg} \cdot \mathrm{min}^{-1}\left(=7.82 \pm 0.7 \mathrm{mU} / \mathrm{kg} \cdot \min ^{-1}\right)$ for $7 \mathrm{~d}$. During the insulin infusion period patients received normal enteral nutrition plus intravenous glucose to maintain euglycemia. In the control period patients received only enteral nutrition. Patients tolerated both the control and insulin plus glucose periods well. There were no episodes of hypoglycemia during insulin infusion. Further, there were no differences during the two periods in $\mathrm{pH}, \mathrm{pO}_{2}, \mathrm{pCO}_{2}$, bicarbonate, AST/ALT, or fibrinogen concentration. In those patients who received ventilatory support, the requirement for support was not affected by the treatment protocol. Since the order of the insulin treatment and control periods was randomized, tracer infusion studies were performed at similar average times after the initial surgery $(20 \pm 3 \mathrm{~d}$ after burn in the control state and $21 \pm 4 \mathrm{~d}$ after burn during insulin infusion).

Plasma glucose and lactate concentrations were essentially constant throughout the isotopic infusion periods of both the control and the insulin plus glucose period. The plasma TG and the VLDL TG concentration were also essentially constant throughout the infusion studies. Similarly, the hematocrit remained unchanged during the two isotope infusion studies and therefore the plasma volume during the same periods was

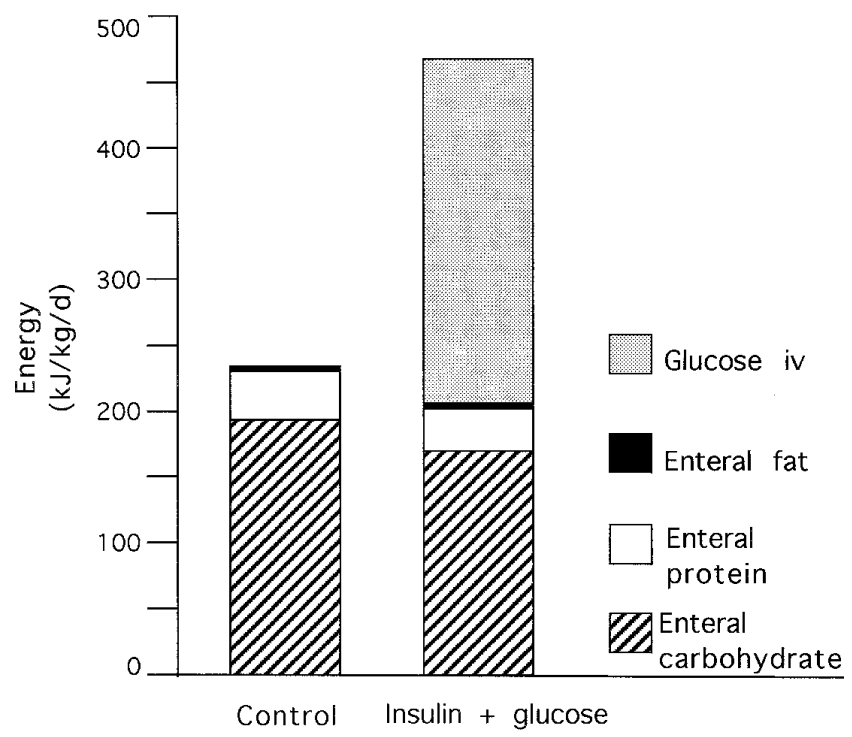

Figure 1. Rate of energy supply. During the 7-d control and the 7-d insulin + glucose period, the patients received a carbohydrate-rich enteral diet providing 1.4 times as many calories as the measured resting energy expenditure. To maintain euglycemia during the insulin treatment additional glucose was infused intravenously at a rate providing $100 \%$ excess calories.

assumed to be unchanged. Consequently, the steady-state conditions required for the equations we used to calculate the results were satisfied.

The body weight of the burned patients increased significantly during the insulin plus glucose treatment, from $64 \pm 16$ $\mathrm{kg}$ in control state, to $67 \pm 15 \mathrm{~kg}$ in the insulin period $(P<$ $0.05)$. Fig. 1 shows that total energy intake was approximately doubled during the insulin therapy, from $242 \pm 71 \mathrm{~kJ} / \mathrm{kg} \cdot \mathrm{d}^{-1}$ in the control period to $468 \pm 138 \mathrm{~kJ} / \mathrm{kg} \cdot \mathrm{d}^{-1}$ during insulin therapy. The extra energy intake during the insulin period was entirely accounted for by the intravenous infusion of glucose that was provided to maintain euglycemia. Caloric intake from the enteral diet (Vivonex ${ }^{\circledR}$ T.E.N.) was similar in the two periods and, thus, the same amounts of protein, fat, and enteral carbohydrates were provided (Table II).

Insulin, glucagon, glucose, and lactate concentrations. Plasma insulin concentration in the control period was $380 \pm 222 \mathrm{pmol} /$ liter and increased during the insulin period to $6,343 \pm 760$ pmol/liter. Plasma glucagon concentrations remained unchanged in the control period and in the insulin period $(310 \pm 135$ and

Table II. Enteral and Parenteral Substrate Provision During the Control and the Insulin plus Glucose Treatment Period

\begin{tabular}{lcc}
\hline & Control & Insulin plus glucose \\
\hline & $\mathrm{g} / \mathrm{kg} \cdot \mathrm{d}^{-1}$ & $\mathrm{~g} / \mathrm{kg} \cdot \mathrm{d}^{-1}$ \\
Enteral carbohydrate & $11.85 \pm 3.42$ & $10.14 \pm 2.93$ \\
Enteral protein & $2.21 \pm 0.55$ & $1.89 \pm 0.55$ \\
Enteral fat & $0.15 \pm 0.04$ & $0.13 \pm 0.04$ \\
Parenteral glucose & 0 & $15.66 \pm 5.28^{*}$
\end{tabular}

$* P<0.05$ statistically different from control. 
Table III. Insulin, Glucagon, Glucose, and Lactate

Concentration in Plasma During the Control and Insulin plus Glucose Treatment Period

\begin{tabular}{lcc}
\hline & Control & Insulin plus glucose \\
\hline Insulin (pmol/liter) & $380 \pm 222$ & $6343 \pm 760^{*}$ \\
Glucagon (ng/liter) & $310 \pm 135$ & $370 \pm 179$ \\
Glucose (mmol/liter) & $7.03 \pm 0.81$ & $5.51 \pm 1.17^{*}$ \\
Lactate (mmol/liter) & $1.47 \pm 0.28$ & $3.58 \pm 1.47^{*}$ \\
\hline
\end{tabular}

$* P<0.05$ statistically different from control period.

$366 \pm 179 \mathrm{ng} /$ liter, respectively). The plasma glucose concentration was significantly lowered during the insulin treatment from $7.03 \pm 0.81 \mathrm{mmol} /$ liter in the control period to $5.51 \pm 1.17$ $\mathrm{mmol} /$ liter in the insulin period (Table III). The plasma lactate concentration increased 2.5-fold during insulin treatment from $1.47 \pm 0.28 \mathrm{mmol} /$ liter in control period to $3.58 \pm 1.47 \mathrm{mmol} /$ liter in the insulin plus glucose period.

Plasma lipids. Insulin plus glucose therapy had no significant effect on the plasma concentration of FA, the plasma palmitate concentration, total plasma TG, or the VLDL TG concentration (Table IV). On the other hand, $7 \mathrm{~d}$ of insulin therapy changed the composition of the VLDL TG-bound FAs (Table V). The relative concentrations of both palmitate (C16:0) and stearate (C18:0) were increased from $35 \pm 5$ and $4 \pm 1 \%$ in the control period to $44 \pm 8$ and $7 \pm 2 \%$ in the insulin period, respectively. The relative concentrations of oleate (C18:1) and linoleate (C18:2) were conversely decreased from $43 \pm 5$ and $8 \pm 4 \%$ before to $37 \pm 6$ and $2 \pm 1 \%$ after insulin therapy respectively. $7 \mathrm{~d}$ of insulin therapy did not change the $\mathrm{Ra}$ FFA in plasma (Table VI). The rate of infusion of labeled acetate was the same during the infusion studies in the control and insulin treatment periods. Nonetheless, the enrichment of the intrahepatic acetyl-CoA pool, i.e., the precursor pool for hepatic de novo FA synthesis, was decreased from $9 \pm 4 \%$ in the control period to $4 \pm 1 \%$ in the insulin period (Table VI). The rate of secretion of de novo-synthesized palmitate bound to VLDL TG was doubled during insulin therapy, but this did not reach statistical significance. The total rate of secretion of VLDL TG bound palmitate was unchanged, while the total rate of secretion of VLDL TG tended to decrease during insulin therapy without reaching statistical significance. The relative amount of de novo-synthesized palmitate out of the total VLDL TG-palmitate (percent de novo-synthesized palmitate) was $13 \pm 5 \%$ in the control period and was significantly increased to $34 \pm 14 \%$ in the insulin plus glucose period (Table VI). Therefore, in the insulin plus glucose period only $66 \pm$

Table IV. Plasma Lipid Concentrations

\begin{tabular}{lcc}
\hline & Control & Insulin plus glucose \\
\hline & $m m o l / l i t e r$ & $m m o l / l i t e r$ \\
Plasma FAs & $0.138 \pm 0.056$ & $0.138 \pm 0.148$ \\
Plasma palmitate & $0.045 \pm 0.018$ & $0.062 \pm 0.074$ \\
Plasma TG & $1.58 \pm 0.66$ & $1.36 \pm 0.41$ \\
VLDL TG & $0.68 \pm 0.79$ & $0.67 \pm 0.63$ \\
& & \\
\hline
\end{tabular}

Table V. Relative FA Concentration in VLDL TG

\begin{tabular}{lrc}
\hline \multicolumn{1}{c}{ FA } & Control & Insulin plus glucose \\
\hline Myristic (C14:0) & $1 \pm 1$ & $3 \pm 3$ \\
Palmitic (C16:0) & $35 \pm 5$ & $44 \pm 8^{*}$ \\
Palmitoleic (C16:1) & $7 \pm 2$ & $8 \pm 2$ \\
Stearic (C18:0) & $4 \pm 1$ & $7 \pm 2^{*}$ \\
Oleic (C18:1) & $43 \pm 5$ & $37 \pm 6^{*}$ \\
Linoleic (C18:2) & $8 \pm 4$ & $2 \pm 2^{*}$ \\
\hline
\end{tabular}

$* P<0.05$ statistically different from control period.

$14 \%$ of the VLDL-bound palmitate was coming from the intrahepatic source of preformed palmitate, while in the control period $87 \pm 5 \%$ of the palmitate was derived from this source (data not shown).

\section{Discussion}

Insulin was administered to severely burned patients at pharmacological doses to promote muscle protein metabolism (10). To maintain euglycemia, glucose was provided along with the insulin at a rate $\sim 100 \%$ in excess of caloric needs. The question addressed in this study was whether the coadministration of pharmacological doses of insulin and glucose would have detrimental effects on the lipid metabolism in the burn patients, in particular, leading to an augmentation of hepatic FA synthesis. This is of concern because steatosis is frequently associated with thermal injury (2-7). Except for the use of repeated liver biopsies the process of fat accumulation in the human liver is inaccessible for direct measurement. This study examined the rate of release of FA from the peripheral tissue, and the hepatic secretion of VLDL TG, both key factors in determining the hepatic fat content. Further, the measured rates of secretion of de novo-synthesized and preformed FA in VLDL TG provide information on the hepatic lipogenic activity.

The starting point in this method is the calculation of the rate of de novo FA synthesis according to the mass isotopomer distribution analysis $(25,26)$. We have recently discussed the details of the calculations and assumptions involved in this approach (27). In the context of this study it is important to emphasize that the method measures the rate of secretion of VLDL-bound lipids, which is not necessarily equal to total hepatic lipogenic activity. During a steady-state one would not expect any net fat accumulation in the liver and, thus, under normal physiological conditions we have used the same approach as a quantitative measurement of hepatic lipid synthesis (14). In this study of burn patients who are possibly retaining hepatic lipids, we compared the rates of secretion of VLDL-bound lipids during two different treatment modalities and therefore did not attempt to quantify the hepatic lipogenic activity. Nonetheless, the rate of secretion of de novo-synthesized FA should provide information about hepatic lipogenic activity, particularly since we used a crossover design.

The rate of incorporation of labeled acetate was only measured in the VLDL-bound palmitate, and based on this FA the total secretion of newly synthesized FA was deduced. We have shown previously that palmitate accounts for $85-90 \%$ of the total hepatic secretion of newly synthesized FA in the basal 


\begin{tabular}{lcc}
\hline & Control & Insulin plus glucose \\
\hline Rate of plasma FA $\left(\mathrm{mmol} / \mathrm{kg} \cdot \mathrm{d}^{-1}\right)$ & $8.22 \pm 2.86$ & $8.72 \pm 6.68$ \\
Precursor enrichment for hepatic FA synthesis $(\mathrm{p})(\%)$ & $9 \pm 4$ & $4 \pm 1^{*}$ \\
Secretion of de novo-synthesized palmitate $\left(\mathrm{mmol} / \mathrm{kg} \cdot \mathrm{d}^{-1}\right)$ & $0.030 \pm 0.03$ & $0.060 \pm 0.06$ \\
Total secretion of VLDL TG palmitate $\left(\mathrm{mmol} / \mathrm{kg} \cdot \mathrm{d}^{-1}\right)$ & $0.181 \pm 0.179$ & $0.177 \pm 0.150$ \\
De novo-synthesized palmitate in VLDL TG palmitate $(\%)$ & $13 \pm 5$ & $34 \pm 14 *$ \\
Total secretion of VLDL TG $\left(\mathrm{mmol} / \mathrm{kg} \cdot \mathrm{d}^{-1}\right)$ & $0.165 \pm 0.138$ & $0.154 \pm 0.138$ \\
\end{tabular}

$* P<0.05$ statistically different from control period.

state and during acute and prolonged lipogenic states (14). In this study as well, stearate (C18) was the only other FA that increased its relative VLDL TG concentration during insulin treatment. To some extent this increase in relative concentration was probably due to increased secretion of newly synthesized stearate (C18). This is supported by the fact that linoleic acid (C18:2) which can only come from the preformed source of FA, decreased in its relative concentration in VLDL TG even though it was provided at a similar rate during both study periods. However, even if $30 \%$ of the secreted stearate (like palmitate) was de novo-synthesized, this source of stearate would only account for $2 \%$ of the total VLDL TGFA secretion, and would only marginally contribute to any difference between the two treatment modalities. With this background we consider the secretion of newly synthesized palmitate as being equivalent to the total secretion of newly synthesized FAbound VLDL TG.

Hepatic lipogenesis could be underestimated by our method if significant amounts of newly synthesized FA entered the circulation in forms other than VLDL TGFA. Consequently, we have determined previously the fractional secretion rate of newly synthesized palmitate in VLDL TGFA, phospholipids, and cholesteryl esters. Secretion of newly synthesized palmitate in phospholipid or cholesterol esters accounted for only $2-3 \%$ of the total secretion of de novo-synthesized palmitate bound to VLDL. There are indications that a minor proportion of the HDL in circulation is secreted directly from the liver (28). The HDL particle is low in TG content but rich in phospholipids. Thus, we believe that HDL secretion from the liver could only be a minor secretory pathway of newly synthesized FA. There is no evidence of hepatic secretion of FFA. Consequently, we can be confident that almost all newly synthesized hepatic FA are secreted in VLDL TG. Furthermore, we have no reason to believe that the relative importance of the VLDL TG as carriers of newly synthesized FA should be affected by the infusion of insulin plus glucose.

Newly synthesized palmitate in this study accounted for only a minor fraction of the total VLDL TG-bound palmitate in the control period $(13 \%)$, but was significantly increased during the insulin plus glucose treatment to account for $34 \%$ of the total VLDL TG-bound palmitate. Out of the total VLDL TGFA secreted, newly synthesized FA accounted for $\sim 5 \%$ in the control and $15 \%$ in the insulin plus glucose period. The relative increase in the concentration of de novosynthesized FA was probably a function of two factors that independently did not reach statistical significance. First, the rate of secretion of newly synthesized FA was doubled during the insulin plus glucose treatment, but did not reach statistical significance. The second factor that contributed to the relative increase of newly synthesized FA in VLDL TG during insulin plus glucose treatment was a decrease in the secretion of preformed FA in VLDL TG. Palmitate which is the predominant product of FA synthesis had its concentration upregulated. Since the relative concentration of palmitate in plasma FA did not change during the insulin plus glucose treatment, this source of FA could not account for the change in palmitate concentration in VLDL TGFA. The only alternative source are the FAs derived from the upregulation of hepatic FA synthesis. The relative concentrations of the FAs coming from the preformed sources oleate (C18:1) and linoleic acid (C18:2) were simultaneously downregulated.

Inasmuch as the relative composition of VLDL TGFA reflects the hepatic lipogenic activity, the results show that an increased carbohydrate load does not represent a detrimental challenge to the hepatic lipid metabolism, provided that euglycemia is maintained. This is clinically the most significant result from this study. The observed shift in relative secretion of de novo-synthesized versus preformed FA should have marginal effects on the hepatic lipid content. Assuming that $1 \mathrm{~g}$ of carbohydrate during lipogenesis yields $0.35 \mathrm{~g}$ of fat (29), only $\approx 50 \mathrm{mg} / \mathrm{kg} \cdot \mathrm{d}^{-1}(<1 \%)$ of the total carbohydrate load could be accounted for by synthesis of VLDL TGFA $\left(15 \mathrm{mg} / \mathrm{kg}^{-1} \mathrm{~d}^{-1}\right)$. Using the same approach one can estimate that out of the 15 $\mathrm{g} / \mathrm{kg} \cdot \mathrm{d}^{-1}$ of the extra glucose that was administered along with the insulin, only $0.3 \%$ was used for de novo synthesis of VLDL TGFA. We have estimated previously, based on an autopsy study, that the liver weight of severely burned patients can increase 1 to $2 \mathrm{~g} / \mathrm{kg} \cdot \mathrm{d}^{-1}$ to reach a weight double that of a normal liver. In a normal liver that is not retaining fat, the secretion of newly synthesized VLDL TGFA would be an estimate of the hepatic lipogenic activity. In a burn patient this is the case only when the liver, in a net sense, is not retaining fat. Still, if the newly synthesized VLDL TGFA during the insulin plus glucose treatment accounted for only $1 \%$ of the total hepatic lipogenic activity and $99 \%$ of the synthesized FA was retained in the liver, then $\sim 1,500 \mathrm{mg} / \mathrm{kg} \cdot \mathrm{d}^{-1}$ de novo-synthesized FA was being deposited in the liver. This extreme example goes to show that the hepatic fat synthesis rate in our study must be underestimated by $99 \%$ to account for the fat that could potentially be deposited in the livers of burn patients. These calculations show that endogenous hepatic fat synthesis can only play a minor role with respect to hepatic steatosis. In addition, carbohydrates provided along with insulin even at a rate two 
times the estimated energy requirements do not change this conclusion.

The fact that the glucose levels were monitored on an hourly basis, and that the glucose infusion was strictly titrated to maintain euglycemia, may explain why hepatic secretion of TGs was only marginally affected by the combined treatment with insulin and high levels of glucose. Insulin-mediated glucose uptake predominantly occurs in muscle and adipose tissue. In contrast, hepatic glucose uptake is an insulin insensitive process governed by the plasma concentration of glucose (30). During the insulin treatment period the plasma glucose concentration was lowered significantly compared to the control period, suggesting that less glucose was taken up by the liver in this period. The majority of the excess glucose was most likely directed to the muscle and the adipose tissue for glycogen and fat synthesis. In healthy volunteers fed a high carbohydrate diet for $4 \mathrm{~d}$ at a rate $150 \%$ in excess of measured energy requirements, we found that hepatic de novo fat synthesis was 0.050 as compared to $0.060 \mathrm{mmol} / \mathrm{kg} \cdot \mathrm{d}^{-1}$ during the insulin plus glucose period in this study. In the healthy volunteers hepatic fat synthesis accounted for no more than $2 \%$ of whole body lipogenesis. If anything, we believe that the hepatic contribution to whole body lipogenesis would be even less during an exogenous insulin infusion.

The concentration of plasma lactate was increased during the insulin plus glucose treatment period. Most likely lactate in excess of hepatic oxidative needs can serve as a substrate for de novo FA synthesis (31) and might explain the slightly stimulated de novo synthesis rate during insulin plus glucose treatment. It is also possible that a simultaneous decrease in glucose and increase in lactate availability causes the liver to channel more preformed FA towards oxidation while simultaneously stimulating de novo FA synthesis. The decreased precursor enrichment for FA synthesis during the infusion of labeled acetate in the insulin plus glucose period suggests that the cytosolic acetyl-CoA pool at this time actually doubled in size.

The high peripheral release of FA during ongoing feeding in the control period is in accordance with our previous measurements of Ra FFA in patients with comparable burn injuries (2). It is reasonable to assume that only a small amount of the released FA was oxidized, since glucose is the preferred energy substrate when both substrates are present $(2,32)$. The high FA release could theoretically provide the equivalent of $\sim 2.3 \mathrm{~g} / \mathrm{kg} \cdot \mathrm{d}^{-1}$ of TG for storage in the liver. Even if only a fraction of the FA release reached the liver, this excess FA release is likely the most important endogenous source of FA for hepatic steatosis. It is striking that a plasma insulin level of $6,343 \pm 760 \mathrm{pmol} /$ liter had no suppressive effect on the high rate of FA release. Apparently, the increased lipolytic activity in association with thermal injury avoids the normal suppressive effect of insulin.

We have shown previously that $7 \mathrm{~d}$ of treatment with pharmacological doses of insulin along with adequate amounts of glucose to maintain euglycemia can reverse a negative muscle protein balance in severely burned patients (10). In this study we conclude that the same treatment regimen only has marginal effects on hepatic lipid production. Even though glucose was provided at a rate $\sim 100 \%$ in excess of estimated energy requirements, the total hepatic VLDL TGFA secretion was not increased, the secretion of de novo-synthesized FA was slightly increased, and the secretion of preformed FA was con- comitantly decreased. The 15 -fold higher plasma level of insulin during the treatment period did not suppress the high peripheral release of FA into plasma compared to the control period. Quantitatively the peripheral release of FA in burn patients represents a far greater potential for hepatic lipid accumulation than the fat being synthesized endogenously in the liver.

\section{Acknowledgments}

The authors would like to thank the nurses and the respiratory therapists at the Burn Unit of The University of Texas Medical Branch and the Shriners Burns Institute in Galveston, Texas, for their assistance. We are indebted to Ms. Yun-xia Lin for her technical assistance.

The study was supported in part by grant 8490 from the Shriners Hospital, NIH grant NK-33952, and grants from The Norwegian Research Council.

\section{References}

1. Wolfe, R.R. 1996. Herman Award Lecture, 1996: relation of metabolic studies to clinical nutrition-the example of burn injury. Am. J. Clin. Nutr. 64: 800-808.

2. Aarsland, A., D. Chinkes, R.R. Wolfe, R.E. Barrow, S.O. Nelson, E. Pierre, and D.H. Herndon. 1996. Beta-blockade lowers peripheral lipolysis in burn patients receiving growth hormone. Rate of hepatic very low density lipoprotein triglyceride secretion remains unchanged. Ann. Surg. 223:777-789.

3. Herndon, D.H., M.D. Stein, T.C. Rutan, S. Abston, and H. Linares. 1987. Failure of TPN supplementation to improve liver function, immunity, and mortality in thermally injured patients. J. Trauma. 27:195-204.

4. Talaat, S.M., G.E. Beheri, M.S. Zaki, and N. El-Bolkainy. 1973. Prevention of early histopathological changes in the liver in extensive burns. Br. J. Plast. Surg. 26:132-139.

5. Wolfe, W.A., J.R. Elkinton, and J.E. Rhoads. 1940. Liver damage and dextrose tolerance in severe burns. Ann. Surg. 112:158-160.

6. James, G.W., O. Purnell, and E.I. Evans. 1951. The anemia of the thermal injury: II. Studies of liver function. J. Clin. Invest. 30:191-199.

7. Burke, J.F., R.R. Wolfe, C.J. Mullany, D.E. Mathews, and D.M. Bier. 1979. Glucose requirements following burn injury. Parameters of optimal glucose infusion and possible hepatic and respiratory abnormalities following excessive glucose intake. Ann. Surg. 190:274-285.

8. Wilmore, D.W., J.A. Moylan, Jr., B.F. Bristow, A.D. Mason, Jr., and B.A. Pruitt. 1974. Anabolic effects of human growth hormone and high caloric feedings following thermal injury. Surg. Gynecol. Obstet. 133:875-884.

9. Revhaug, A. and M. Mjaaland. 1993. Growth hormone and surgery. Horm. Res. 40:99-101.

10. Sakurai, Y., A. Aarsland, D.N. Herndon, D.L. Chinkes, E. Pierre, T.T. Nguyen, B.W. Patterson, and R.R. Wolfe. 1995. Stimulation of muscle protein synthesis by long-term insulin infusion in severely burned patients. Ann. Surg. 222:283-297.

11. Chascione, C., D.H. Elwyn, M. Davila, K.M. Gil, J. Askanazi, and J.M. Kinney. 1987. Effect of carbohydrate intake on de novo lipogenesis in human adipose tissue. Am. J. Physiol. 253:E664-E669.

12. Guenst, J.M., and L.D. Nelson. 1994. Predictors of total parenteral nutrition-induced lipogenesis. Chest. 105:553-559.

13. Hill, J.O., and A.M. Prentice. 1995. Sugar and body weight regulation. Am. J. Clin. Nutr. 62(Suppl.):S264-S274.

14. Aarsland, A., D. Chinkes, and R.R. Wolfe. 1997. Hepatic and wholebody fat synthesis in humans during carbohydrate overfeeding. Am. J. Clin. Nutr. 65:1774-1782.

15. Coppack, S.W., M.D. Jensen, and J.M. Miles. 1994. In vivo regulation of lipolysis in humans. J. Lipid Res. 35:177-193.

16. Woodside, W.F., and M. Heimberg. 1976. Effects of anti-insulin serum, insulin, and glucose on output of triglycerides and ketogenesis by the perfused rat liver. J. Biol. Chem. 251:13-23.

17. Fukuda, N., and J.A. Ontko. 1984. Interactions between fatty acid synthesis, oxidation, and esterification in the production of triglyceride-rich lipoproteins by the liver. J. Lipid Res. 25:831-842.

18. Aarsland, A., D. Chinkes, and R.R. Wolfe. 1996. Contributions of de novo synthesis of fatty acids to total VLDL-triglyceride secretion during prolonged hyperglycemia/hyperinsulinemia in normal man. J. Clin. Invest. 98:20082017.

19. Lewis, G.F., K.D. Uffelman, L. Szeto, B. Weller, and G. Steiner. 1995. Interaction between free fatty acids and insulin in the acute control of very low density lipoprotein production in humans. J. Clin. Invest. 95:158-166.

20. Gibbons, G.F. 1989. Insulin, diabetes and hepatic very-low-density lipo- 
protein metabolism. Biochem. Soc. Trans. 17:49-51.

21. Grundy, S.M., H.Y.I. Mok, L. Zech, and D. Steinberg. 1979. Transport of very low density lipoprotein triglycerides in varying degrees of obesity and hypertriglyceridemia. J. Clin. Invest. 63:1274-1283.

22. McGowman, M.W., J.D. Artiss, D.R. Strandbergh, and B. Zak. 1983. A peroxidase-coupled method for the colorimetric determination of serum triglycerides. Clin. Chem. 29:538-542.

23. Wolfe, R.R. 1992. Radioactive and Stable Isotope Tracers in Biomedicine: Principles and Practice of Kinetic Analysis. Wiley-Liss, New York. 317-340.

24. Armitage, P., and G. Berry. 1994. Statistical Methods in Medical Research. Blackwell Scientific Publications, Oxford. 112-120.

25. Hellerstein, M.K., C. Kletke, S. Kaempfer, K. Wu, and C.H. Shackleton. 1991. Use of mass isotopomer distributions in secreted lipids to sample lipogenic acetyl-CoA pool in vivo in humans. Am. J. Physiol. 261:E479-E486.

26. Hellerstein, M.K., and R.K. Neese. 1992. Mass isotopomer distribution analysis: a technique for measuring biosynthesis and turnover of polymers. Am. J. Physiol. 263:E988-E1001.
27. Chinkes, D., A. Aarsland, J. Rosenblatt, and R.R. Wolfe. 1996. A comparison of mass isotopomer dilution methods used to compute production of VLDL fatty acids in vivo in human subjects. Am. J. Physiol. 271:E373-E383.

28. Glickman, R.M., and S.M. Sabesin. 1994. Lipoprotein metabolism. In The Liver, Biology and Pathobiology. I.M. Arias, J.L. Boyer, N. Fausto, W.B Jakoby, D. Schachter, and D.A. Shafritz, editors. Raven Press, New York. 391-414 29. Ferrannini, E. 1988. The theoretical bases of indirect calorimetry: a review. Metabolism. 37:287-301.

30. DeFronzo, R.A. 1988. The triumvirate: b-cell, muscle, liver. A collusion responsible for NIDDM. Diabetes. 37:667-687.

31. Newton, R.S., and R.A. Freedland. 1980. The effects of specific lipogenic substrates and metabolic inhibitors on de novo fatty acid synthesis in isolated hepatocytes from chow-fed female rats. Arch. Biochem. Biophys. 204: 379-386.

32. Wolfe, B.M., S. Klein, E.J. Peters, B.F. Schmidt, and R.R. Wolfe. 1988 Effect of elevated free fatty acids on glucose oxidation in normal humans. $M e$ tabolism. 37:323-329. 